of the brain vessels etc. Patients with rheumatic diseases also have an elevetaion of endothelin-1 in serum. But it remains unclear whether the endothelin-1 level may reflect endothelium dysfunction in patients with antiphospholipid syndrome (APS) and serve as an early marker of atherosclerosis.

Objectives: The aim of the study was to evaluate the endothelin-1 concentration, its association with antiphospholipid antibodies and atherosclerotic vascular lesions in patients with APS.

Methods: According to our observation, there were 82 patients, among which 34 $(41.6 \%)$ with primary antiphospholipid syndrome (PAPS) and $48(58.4 \%)$ with secondary antiphospholipid syndrome (SAPS). The control group consisted of 37 practically healthy persons. The groups of patients were comparable by age, sex, and duration of the disease. The APS diagnosis was established according to the international classification criteria (2006). The content of the anticardiolipin antibodies IgG, anti-beta2-glycoprotein-1 antibodies $\lg G$, IgA, IgM and endothelin-1 were determined by the immune enzyme method using commercial sets of Trinity Biotech Captia USA - Ireland, ORGenTec GmbH Germany and «Endothelin-1» (Cormay, England). All patients were assessed for the level of endotheliumdependent vasodilation (EDVD), the thickness of the intima-media complex of the common carotid artery (IMT), the presence of atherosclerotic plaque (AP) and clinical manifestations of vascular involvement.

Results: The analysis of endothelin-1 levels has showed that its content was in 2.3 times higher in patients with APS than in the control group. The proportion of people with optimal endothelin-1 content was twice lower, and the proportion of people with extremely high and high rates was 1.6 and 5.3 times higher, respectively, among patients with APS, than in the control group. Also differences in the endothelin-1 level have been established depending on the type of APS. The content of endothelin-1 was significantly higher (1.3 times) in patients with SAPS, than in PAPS. Among the patients with SAPS, the proportion of patients with an optimal level of endothelin-1 was 1.7 times lower, and the proportion of patients with high levels was 1.6 times higher than in patients with PAPS. The significantly lower endothelin-1 level was recorded in patients with highly positive antibodies to cardiolipin and beta-2 glycoprotein-1. Correlation analysis has showen direct correlation between anticardiolipin antibodies of the IgG class and anti-beta2-glycoprotein-1 antibodies and endothelin-1 concentration ( $r=0.35$ and 0.34$)$. High endothelin-1 level was an adverse factor of structural and functional atherosclerotic changes of the heart and blood vessels in patients with APS. IMT increasing and EDVD decreasing were from 1.7 to 6.6 times more frequent among patients with high endothelin-1 level (>10 pg/ml), than patients with optimal endothelin-1 level $(\leq 5.0 \mathrm{pg} / \mathrm{ml})$.

Conclusion: Thus, the obtained data has showed that the excess concentration of endothelin-1 is a circulating marker of early atherosclerosis, since it is closely associated with subclinical manifestations of atherosclerotic vascular damage

Disclosure of Interests: None declared

DOI: 10.1136/annrheumdis-2019-eular.2990

\section{AB0192 ASYMPTOMATIC VERTEBRAL FRACTURES WORSEN ORGAN DAMAGE IN WOMEN WITH SYSTEMIC LUPUS ERYTHEMATOSUS}

Svetlana Shkireeva ${ }^{1}$, Olga Lesnyak ${ }^{2}$, Evgeniy Zotkin ${ }^{1} .{ }^{1}$ Research Institute of Rheumatology named after V.A.Nasonova, Moscow, Russian Federation; ${ }^{2}$ NorthWestern State Medical University named after I.I.Mechnikov, Saint-Petersburg, Russian Federation

Background:

Objectives: To investigate the influence of asymptomatic vertebral fractures on organ damage and to identify risk factors associated with critical organ damage in women with systemic lupus erythematosus (SLE)

Methods: 207 women with SLE were included in this study. Bone mineral density (BMD) measurements of the hip and spine were performed using the dual energy X-ray absorptiometry (DXA). Vertebral fracture assessment (VFA) was done for detection vertebral fractures using a method described by Genant. Accumulated damage was scored using the SLICC/ACR damage index (SDI). Critical organ damage was defined as $\mathrm{SDI}>3$.

Results: Vertebral fractures were developed in 55 (26.5\%) women with SLE. Half of all women with SLE ( $n=31,15.0 \%)$ had asymptomatic vertebral fractures which were diagnosed for the first time in this study. 131 (63.3\%) women with SLE had critical organ damage $(\mathrm{SDI}>3)$. Average $\mathrm{SDI}$ before and after morphometry was $4,5 \pm 2,1$ and $5,4 \pm 2,5$ respectively. Multivariate analysis showed age $(p=0,01)$ and cumulative dose of glucocorticoids $(p=0,00005)$ were significantly associated with critical damage in women with SLE.

Conclusion: Detection of vertebral fractures helps in counting accumulated organ damage correctly. VFA in the combination with DXA in women with SLE is an effective method for diagnostic asymptomatic vertebral fractures.

Disclosure of Interests: None declared

DOI: 10.1136/annrheumdis-2019-eular.3058

\section{$\mathrm{AB} 0193$ \\ THE EFFECTS OF NON-INVASIVE VAGUS NERVE STIMULATIONON FATIGUE AND NEUROCOGNITION IN PARTICIPANTS WITH PRIMARY SJÖGREN'S SYNDROME}

Jessica Tarn ${ }^{1}$, Victoria Macrae ${ }^{2}$, Sheryl Mitchell ${ }^{2}$, Bruce Simon ${ }^{3}$, Peter Gallagher ${ }^{1}$, John-Paul Taylor ${ }^{1}$, Stuart Watson ${ }^{1}$, Mark Baker ${ }^{1}$, Stephen Rushton ${ }^{1}$,

Andrew Blamire ${ }^{1}$, Julia Newton ${ }^{1}$, Wan Fai $\mathrm{Ng}^{1}$. ${ }^{1}$ Newcastle University, Newcastle upon Tyne, United Kingdom; ${ }^{2}$ Newcastle upon Tyne Hospitals NHS Foundation Trust, Newcastle upon Tyne, United Kingdom; ${ }^{3}$ electroCore, Basking Ridge, NJ, United States of America

Background: Primary Sjögren's syndrome (pSS) sufferers have rated physica and mental fatigue ('brain fog') as the most important symptoms needing improvement. Previous data from our group suggest that stimulation of the vagus nerve can invoke immunological responses and concurrently an improvement of patient reported symptoms of fatigue ${ }^{1}$

Objectives: This follow up study uses the gammaCore device (electroCore) and a sham device to assess the effects of non-invasive vagus nerve stimulation (nVNS) on patient reported symptoms of fatigue in pSS. In addition, neurocognitive tests were used to assess the effect of nVNS on short term memory, executive function and attention.

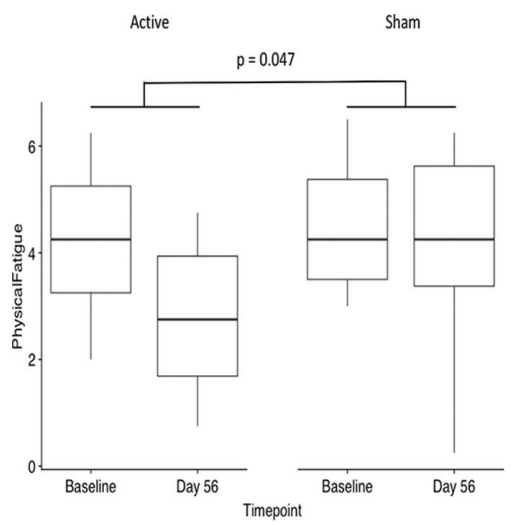

Figure 1

Methods: 40 pSS participants were assigned to use active $(n=20)$ or sham $(n=$ 20) nVNS devices. Participants and research staff were blinded to the active/ sham device assignment. The participants were instructed to use the device twice daily for a study period of 56 days. The following patient reported measures of fatigue and neuropsychological tests were collected at baseline and day 56; Profile of Fatigue (PRO-F, Physical and Mental), visual analogue scale (VAS) of abnormal fatigue, trail making, Digit Symbol Substitution Test (DSST), Stroop test and digit span. 7 subjects were excluded from the analysis due to withdrawal from the study. Changes in fatigue and test scores from baseline to day 56 were com pared between devices using t-tests.

Results: Physical fatigue was significantly reduced between baseline and day 56 in the active group but not the placebo group $(p=0.047)$ The mean reduction in physical fatigue was $30 \%$ and $6 \%$ for the active and sham arms respectively (Figure 1). In both the active and sham subject groups there were no significan changes in the patient reported mental fatigue or neuropsychological test scores. Conclusion: This sham controlled scientific study of vagus nerve stimulation in pSS suggests nVNS may improve patient reported symptoms of physical fatigue which is consistent with our published data. There were no improvements in the neurosychological test scores which is consistent with no improvements in patient reported mental fatigue. nVNS has been shown to activate the anti-inflammatory reflex, therefore assessment of peripheral inflammatory markers may help our understanding of nVNS, inflammation and fatigue.

\section{REFERENCE}

[1] Tarn, J., Legg, S., Mitchell, S., Simon, B. \& Ng, W.-F. The Effects of Noninvasive Vagus Nerve Stimulation on Fatigue and Immune Responses in Patients With Primary Sjögren's Syndrome. Neuromodulation 49, 844 (2018).

Disclosure of Interests: Jessica Tarn: None declared, Victoria Macrae: None declared, Sheryl Mitchell: None declared, Bruce Simon Shareholder of: Bruce Simon is an employee and shareholder of electroCore., Peter Gallagher: None declared, John-Paul Taylor: None declared, Stuart Watson: None declared, Mark Baker: None declared, Stephen Rushton: None declared, Andrew Blamire: None declared, Julia Newton: None declared, Wan Fai Ng: None declared DOI: 10.1136/annrheumdis-2019-eular.8046 